The Planetary System, Apollo 11 at 25, Scientific Results from the Goddard High Resolution Spectrograph, The Impact Catastrophe that Ended the Mesozoic Era, and some other terrestrial subjects. They are available as computer disks or as a CDROM that includes them all. They can also be downloaded over the Internet. Get information from clallo@stsci.edu.

7. Astronomical Society of the Pacific. The Astronomical Society of the Pacific (390 Ashton Avenue, San Francisco, CA 94122, fax 415-337-5205, Internet: asp@stars.sfsu.edu) is a supplier of a wide variety of electronic media resources, including computer software, CD-ROM's and videodiscs.

8 . World Wide Web. The Internet has brought almost instant access to scientific data to many sites around the world. For example, the morning after observations were taken of the impact of Comet Shoemaker-Levy 9 on Jupiter, many observations from major observatories were posted on the Internet. Use of a graphics interface like Mosaic, and calling the Jet Propulsion Laboratory's "News Flashes" on www.jpl.nasa.gov, brought a choice of dozens of images via the World Wide Web.

\title{
RADIO ASTRONOMY SYSTEMS FOR UNDERGRADUATES
}

\author{
Aa. Sandqvist \\ Stockholm Observatory, S-133 36 Saltsjöbaden, Sweden \\ E-mail: sandqvis@astro.su.se \\ and \\ M. Hagström \\ Onsala Space Observatory, S-439 92 Onsala, Sweden
}

1. Introduction. In Sweden, there are four university departments of astronomy (Gothenburg, Lund, Stockholm and Uppsala) and one National Facility for Radio Astronomy, namely Onsala Space Observatory (OSO). Radio astronomy courses are offered only at OSO, which also hosts annual study visits by the country's advanced undergraduates in astronomy.

Teaching observational astronomy in Sweden has always been severely hampered by its high northern latitude and by its coastal climate. The light summer nights makes optical astronomy impossible during this season, and the large percentage of clouds and its unpredictability throughout the year almost annihilates organized class observational exercises.

As is well known, radio astronomical observations are possible both during the day and the night, as well as during cloudy conditions. It is therefore possible to plan schedules for undergraduate radio astronomy observing sessions without worrying about the weather or the time of day. "All" you need is the hardware and the software. We have therefore initiated a project for the construction of seven radio 
telescopes and receivers to be distributed at different Swedish Universities and Institutes of Technology. Physics Departments at Stockholm University and the Royal Institute of Technology are also obtaining these radio astronomy systems, so that physics students may come into early contact with modern astronomical techniques.

2. Mass Production. The radio telescopes and receivers are being designed and constructed at OSO. We have the main responsibility for the project, but three other persons are involved to a large extent, namely $P$. Hillerström (microprocessor and correlator), A. Kristenson (servo-motor drive card) and M. Olberg (software).

Mass production of such systems has now become realistic, thanks to new technology which makes it possible to obtain relatively inexpensive "off-the-shelf" items. For example, the great variety of satellite TV dishes on the market yields the reflectors and elevation drive motors for the radio telescopes. There also exists an ample supply of GaAs amplifiers and other integrated solid state devices. On the other hand, a large amount of design and developmental work has been carried out in connection with the telescope mounts, encoders, azimuth drive motors, and receivers. Personal computers can be used for telescope control, data acquisition and data analysis, once the software has been created. For the spectral line receiver, we are at a convenient time for this project. OSO has recently ordered one thousand 16channel correlator chips for a multi-channel hybrid correlator to be used in a multibeam imaging system being designed for the OSO $20-\mathrm{m}$ millimetre wave telescope. We get 21 of these chips for our program.

3. System Parameters. Each radio telescope consists of a $2.3 \mathrm{~m}$ parabolic reflector with a dipole feed mounted on a central leg. The telescope can be moved in azimuth $\left(-199^{\circ}\right.$ to $\left.+199^{\circ}\right)$ and elevation $\left(+5^{\circ}\right.$ to $\left.+85^{\circ}\right)$. The half-power beamwidth is approximately $7^{\circ}$.

The $1420 \mathrm{MHz}$ continuum receiver has a bandwidth of $50 \mathrm{MHz}$. The $21 \mathrm{~cm} \mathrm{H}$ I line receiver is tunable within this bandwidth and has an instantaneous bandwidth of $2 \mathrm{MHz}$. Both load-switching and frequency-switching modes of operation are possible. The system temperature is of the order of $200 \mathrm{~K}$ and the temperature of the calibrator is $26 \mathrm{~K}$.

The spectrum analyzer consists of a 48-channel correlator with a total usable bandwidth of $2 \mathrm{MHz}$. This corresponds to a channel frequency separation of $50 \mathrm{kHz}$, or $10.5 \mathrm{~km} / \mathrm{s}$ per channel in radial velocity. There is free choice of integration times, but a one-minute integration suffices to obtain a high-quality $\mathrm{H}$ I profile.

The computer is a Personal Computer of the type IBM 386 (minimum IBM 286), or IBM compatible, with TURBOPASCAL. The PC has two main functions: telescope and receiver interactions, and continuum and spectral line data analysis. The former function sends commands to the microprocessor for steering the telescope and receiver parameters, and receives the continuum and line data from the receivers and stores these on the PC hard disk. Spectal line data analysis is performed with DRAWSPEC, a spectral line processing system developed for IBM-compatible PCs by Harvey Liszt at the US National Radio Astronomy Observatory.

4. Conclusion. Three radio telescope systems are now in operation, one at Stockholm Observatory (which is the Institute of Astronomy at Stockholm University), one at the Department of Physics at Stockholm University, and one at OSO (which is an 
institute of Chalmers University of Technology in Gothenburg as well as the Swedish National Facility for Radio Astronomy). Future systems will soon be operating at the Institute of Astronomy at Lund University, the Department of Physics at the Royal Institute of Technology in Stockholm, the Institute of Astronomy at Uppsala University, and finally, a second system at OSO. It is hoped that many future astronomy and physics undergraduate students will be stimulated by using these radio telescope systems for their own personal interaction with the wonders of the sky.

THE CCD IN UNDERGRADUATE PRACTICAL ASTRONOMY

D. McNally

University of London Observatory, Mill Hill Park, London NW7 2QS, UK

1. Introduction. The CCD is producing a revolution in astronomy at the end of the 20th century, equivalent to the photographic revolution at the end of the 19th century. That revolution spread from the major international observatory right through the university observatory to amateur astronomy. Small telescopes, previously considered too small or too poorly situated for worthwhile work are now empowered in a way that was undreamt of surprisingly few years ago. With exposure times in seconds and minutes where previously they were in hours, it means that those of us who do not enjoy the best of climates (or weather) can now utilise fleeting half hours of clearish skies. The response of CCDs also means that for those of us who "enjoy" superb bright skies at night, imaging of extended objects is possible once again. It is not surprising that CCDs have been introduced into the undergraduate astronomy practical class.

2. CCDs at the University of London Observatory. I had wished to introduce CCD detectors at the University of London Observatory from about 1985. Apart from natural reluctance of colleagues to abandon the classic astronomical plate, there were two reasons why our entry into this field was delayed - cost and the opinion that undergraduates and cryogenic fluids did not mix. Declining costs and the development of Peltier cooled CCD detectors removed those two obstacles.

The problem to be faced was what criteria should be applied to selecting a CCD for undergraduate use. Very clearly professional quality CCDs were still beyond our financial grasp, yet if students were to be introduced to CCD based working, the system could not be a toy. A survey of the market in 1989-90 revealed that a local firm (Wright Instruments Ltd., Enfield) was offering a $600 \times 400$ pixel detector, Peltier cooled to $70^{\circ} \mathrm{K}$ below ambient. The price was not cheap but with software and dedicated PC the bill was of the order of $£ 20,000$. We went ahead and mounted the system for the Observatory's spectral classification spectrograph designed by M.M. Dworetsky. The CCD camera may also be used for imaging. It was at once clear that we had a winning combination.

A second large format $(1200 \times 800$ pixel $) \mathrm{CCD}$ was obtained from the same 